\title{
CALIBRATION OF BEAM POSITION MONITORS USING A STORED BEAM IN THE SPring-8 STORAGE RING
}

\author{
K. Kumagai, N. Kumagai, H. Ohkuma, K. Soutome* ${ }^{*}$ M. Takao, H. Tanaka \\ SPring-8, Mikazuki, Sayo, Hyogo 679-5198, Japan
}

\begin{abstract}
A simple and practical method is proposed to estimate offsets of beam position monitors. It is reported that this method had been applied successfully to an $8 \mathrm{GeV}$ electron storage ring at SPring-8. Resulting offsets are compared with those measured by using a beam-based method.
\end{abstract}

\section{INTRODUCTION}

In particle accelerators, such as electron or positron storage rings, calibration of beam position monitors (BPMs) is an important subject for achieving a design performance of the beam quality. To eliminate systematic errors, or the offsets of BPMs, a beam-based method is generally useful[1], [2], [3]. This method, however, requires additional power supplies to quadrupole magnets and can not be applied to the whole ring immediately.

At the SPring- 8 storage ring, a high quality of the beam is also required: high stability of an orbit, a small beam emittance, a small coupling ratio of horizontal and vertical betatron oscillations, etc. Since the total number of quadrupole magnets is 480 and only 40 of them can change their strength independently, all 288 BPMs in the ring can not be calibrated by the beam-based method.

We then estimated BPM offsets in a simple and practical manner by using closed orbit data[4]. In the following we explain how we carried out this and improved the quality of a stored beam.

\section{METHOD}

A part of BPM offsets can be estimated by summing Fourier components of COD whose harmonic number is much higher than betatron tunes. Such high harmonic components of the offsets should be eliminated in orbit corrections, since main magnets are usually aligned so that their magnetic centers are connected as smoothly as possible. At the SPring-8 storage ring, for example, the concept of "two-stage magnet alignment with common girders" was introduced[5] and main magnets were aligned carefully with a laser-alignment system with the accuracy of less than $20 \mu m[6]$.

The procedure for obtaining the "offsets" is as follows:

\footnotetext{
*Corresponding author; Email: soutome@ spring8.or.jp
}

- Store the beam and measure the closed orbit with BPMs. If necessary, make a rough orbit correction with a small number of steering magnets to prevent nonlinear behaviors of the BPM system. If possible, select an operation point with a small integer part of the betatron tunes so that low harmonic components coming from real orbit distortions and high harmonic components coming from BPM "offsets" are separated as clearly as possible.

- Make a Fourier decomposition of the orbit data as follows:

$$
\begin{gathered}
\frac{\operatorname{COD}(s)}{\sqrt{\beta(s)}}=\sum_{n=0}^{\infty}\left(a_{n} \cos n \phi(s)+b_{n} \sin n \phi(s)\right) \\
\phi(s) \equiv \int_{0}^{s} \frac{d s^{\prime}}{\nu \beta\left(s^{\prime}\right)}
\end{gathered}
$$

where $\beta(s)$ is the betatron function at the position $s$ and $\nu$ is the betatron tune.

- Reconstruct the orbit by collecting Fourier components whose harmonic number is smaller than some cutoff value. On determining the cutoff value we will discuss in the next section.

- Subtract the reconstructed orbit from a measured orbit to obtain the "offsets".

\section{APPLICATION TO THE SPring-8 STORAGE RING}

We note that the basic lattice structure of the SPring- 8 storage ring is of the double-bend achromat (DBA) type with 48 unit cells. In each unit cell there are 6 BPMs (BPM1, BPM2, ..., BPM6) located in such a way that the phase difference of betatron oscillations between BPM1 and BPM2, BPM3 and BPM4, and BPM5 and BPM6 becomes small. Since each pair of these BPMs are welded on the same vacuum chamber and all nearby quadrupole and sextupole magnets are well aligned on the same girder, the difference of beam positions measured by each pair should be small. Then, we can expect that spurious spikes of the position data, or harmful BPM offsets, can be distinguished by the method described in the previous section.

Before applying this method we must determine the cutoff harmonic number so that the contribution from higher 
harmonic components of a real orbit distortion is much smaller than that of spurious BPM offsets. With the help of computer simulations we determined the cutoff harmonic number in the following way:

- Calculate a closed orbit by using alignment data and field strengths of main magnets measured beforehand. The result simulates an orbit without corrections.

- Make a Fourier decomposition of the calculated orbit and find a cutoff harmonic number above which the rms value of the sum of higher harmonic components becomes smaller than some tolerance. In the SPring- 8 storage ring this toralence was set to be $50 \mu \mathrm{m}$.

As can be seen from the above procedure, the cutoff value depends on the betatron tunes of the ring. Note also that in order to separate components coming from real orbit distortions and those from spurious BPM offsets as clearly as possible, it is better to lower the betatron tune. This can be seen from the following formula:

$$
\frac{\operatorname{COD}(s)}{\sqrt{\beta(s)}}=\sum_{m=-\infty}^{\infty} \frac{\nu^{2}}{\nu^{2}-m^{2}} f_{m} e^{i m \phi(s)}
$$

where

$$
f_{m} \equiv \frac{1}{2 \pi \nu} \sum_{i} \sqrt{\beta\left(s_{i}\right)} \theta_{i} e^{-i m \phi\left(s_{i}\right)}
$$

and $\theta_{i}$ is the kick at the position $s_{i}$.

In the SPring-8 storage ring we examined five different optics with the following horizontal and vertical betatron tunes: $\left(\nu_{H}, \nu_{V}\right)=(51.23,16.32),(42.20,15.32)$, $(42.24,12.21),(21.35,9.17)$ and $(18.25,13.69)$. The last three optics with low betatron tunes are not of the standard DBA type. These were designed especially for checking the optics dependence. In Fig. 1 we show the rms difference of full and reconstructed CODs as a function of the cutoff harmonic number for the optics with tunes (51.23, $16.32),(42.20,15.32)$ and $(21.35,9.17)$. The cutoff values for these optics were determined from the saturation profile of this rms difference to be $(150,40),(150,40)$ and $(70,40)$, respectively[7].

In Fig. 2 we show BPM "offsets" obtained experimentally by using the above three optics. We see that the optics dependence of the "offsets" is very weak, especially for the vertical direction. The rms value of the "offsets" for these optics differs only by $40 \mu \mathrm{m}$ in the horizontal direction and $10 \mu \mathrm{m}$ in the vertical direction. Then we can conclude that a dominant and harmful contribution to the "offsets" was obtained by the present method.

To go ahead with orbit corrections, we used the "offsets" for the optics with betatron tunes $(21.35,9.17)$ which are indicated by the solid curve in Fig. 2. The rms values of the "offsets" is $160 \mu \mathrm{m}$ in the horizontal direction and $210 \mu \mathrm{m}$ in the vertical direction.

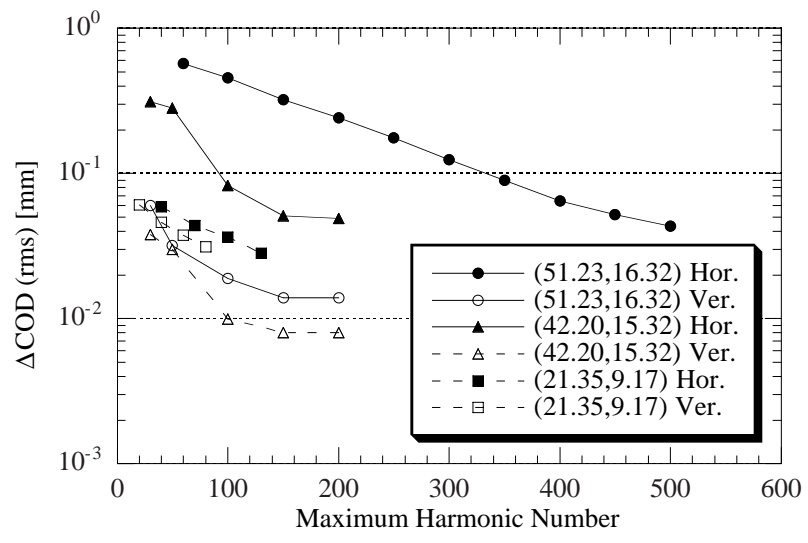

Figure 1: The rms difference of full and reconstructed CODs.
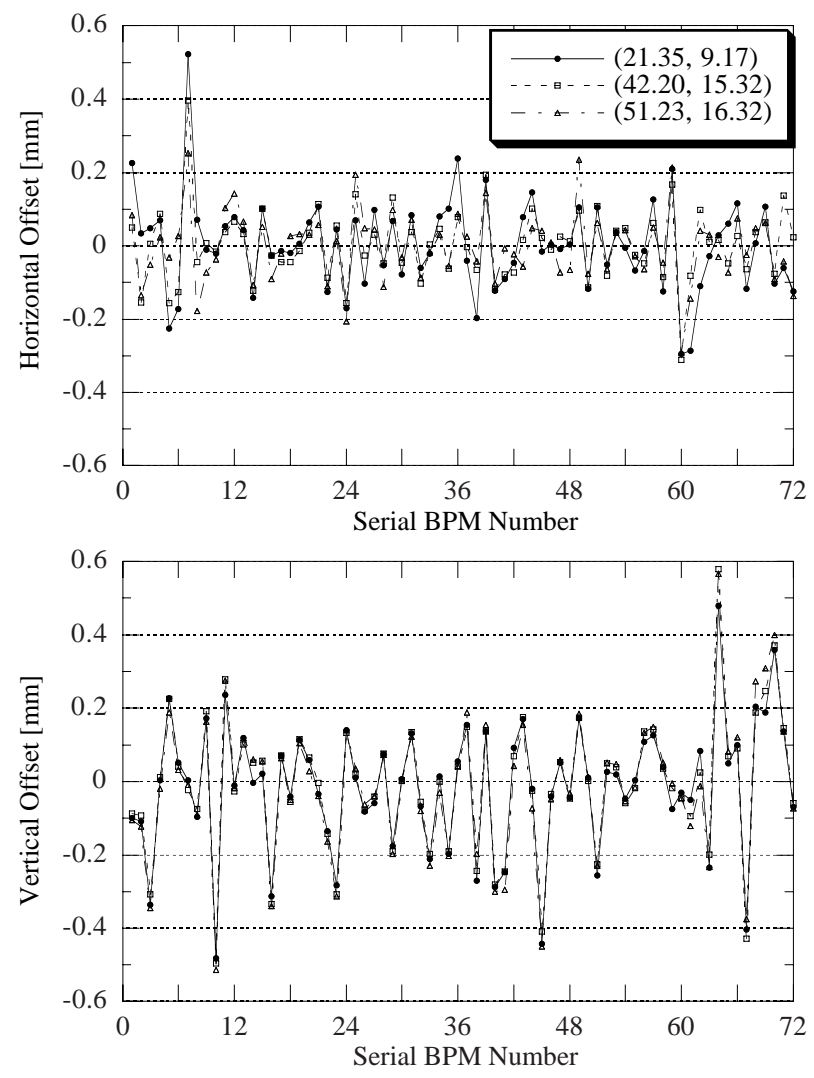

Figure 2: BPM "offsets" in a quarter of the ring obtained experimentally with three different optics in the horizontal (above) and vertical (below) directions.

\section{COMPARISON TO BEAM-BASED METHOD}

To check the reliability of the "offsets", we measured a beam position at some BPMs with respect to the center of a nearby quadrupole magnet by using a beam-based method. An example is shown in Fig. 3, where a magnetic center of a target quadrupole magnet is searched by changing its 
strength and detecting the amplitude of an induced betatron oscillation. The technique used is essentially the same as those in [1], [2], [3], but our method is different in the point that (i) a single kick is used instead of a local bump to generate a parallel shift of an orbit at a target quadrupole magnet and (ii) a Fourier component corresponding to the betatron tune is plotted.

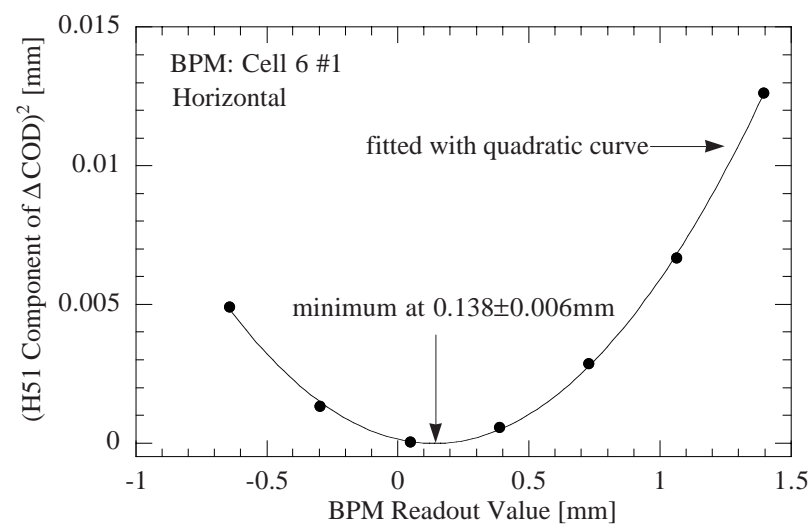

Figure 3: An example of beam-based measurement of a BPM offset.

With this method the offsets were measured at 24 BPMs. The results are shown in Fig. 4 by dashed curves. Also shown by solid curves are the corresponding "offsets" obtained from high harmonic Fourier components. The agreement between the solid and dashed curves is not perfect but satisfactory. We see that they have very similar tendencies. The rms value of the difference is about $150 \mu \mathrm{m}$ in both horizontal and vertical directions. This value is a measure of the amount of remaining low harmonic components of the BPM offsets.

\section{CONCLUSION}

After calibrating BPMs by using the "offsets" shown in Fig. 2, the quality of a stored beam has been improved. For example, we could further reduce COD by using steering magnets with weak strengths. The leakage of the dispersion function into straight sections also reduced from about $23 \mathrm{~mm}$ to $12 \mathrm{~mm}$ in rms values. We note here that the coupling ratio of the horizontal and vertical betatron oscillations has been deduced from Touschek lifetime and found to be very small [8]: much less than $1 \%$ without corrections by skew quadrupole magnets.

All of these facts indicate that the electron beam passes through the position close to magnet centers all along the ring and hence the BPM "offsets" shown in Fig. 2 worked well in orbit corrections.

Acknowledgment: The authors would like to thank Drs. S.Daté, K.Fukami, M.Masaki, T.Ohshima, S.Sasaki, M.Shoji, S.Takano and K.Tamura for useful discussions and cooperation in machine operations.
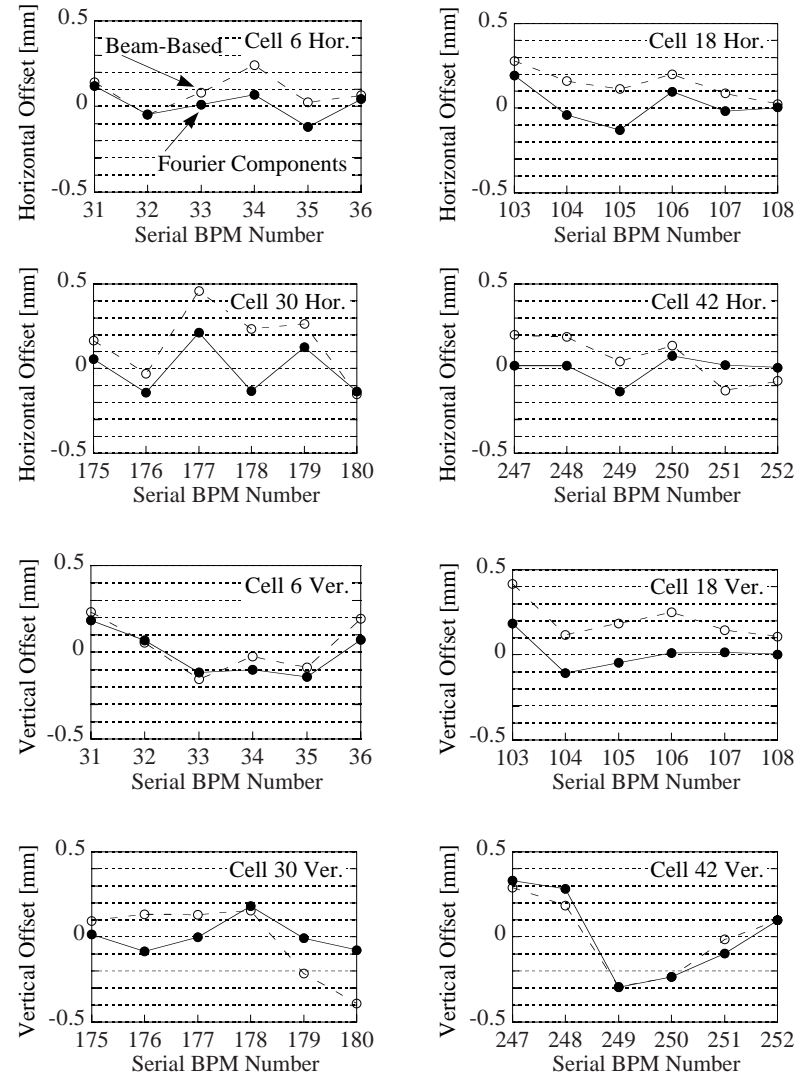

Figure 4: Comparison of BPM offsets obtained by two different methods.

\section{REFERENCES}

[1] See e.g., Proc. 4th Int. Workshop on Accelerator Alignment (KEK Proceedings 95-12), Tsukuba, 14-17 November 1995, chapter V.

[2] P. Röjsel, Nucl. Instr. and Meth. A343(1994)374.

[3] B. Dehning, G. -P. Ferri, P. Galbraith, G. Mugnai, M. Placidi, F. Sonnemann, F. Tecker and J. Wenninger, Proc. 6th European Particle Accelerator Conf., Stockholm, 22-26 June 1998, p.430.

[4] H. Tanaka, K. Soutome, M. Takao, H. Ohkuma and N.Kumagai, SPring-8 Ann. Rep. 1997, p.188.

[5] H. Tanaka, N. Kumagai and K. Tsumaki, Nucl. Instr. and Meth. A313(1992)529.

[6] K. Tsumaki, N. Kumagai, S. Matsui, C. Zhang and J. Ohnishi Proc. 6th European Particle Accelerator Conf., Stockholm, 22-26 June 1998, p.1356.

[7] For the optics $(51.23,16.32)$ the rms difference in the horizontal direction converges very slowly with the maximum harmonic number. We thus set the cutoff value to be 150 and used the resulting "offsets" only for comparison to other cases.

[8] M. Takao, et.al., "Estimation of Betatron Coupling and Vertical Dispersion for SPring-8 Storage Ring", presented at this conference. 